\section{Intentional passive eruption combined with scaling and root planing of teeth with moderate chronic periodontitis and traumatic occlusion}

\author{
Ji-Young Joo, Eun-Young Kwon, Ju-Youn Lee* \\ Department of Periodontology, Pusan National University School of Dentistry, Yangsan, Korea
}

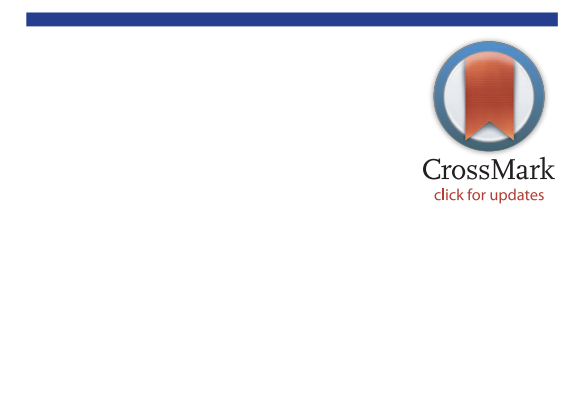

\section{Research Article}

J Periodontal Implant Sci 2014;44:20-24

http://dx.doi.org/10.5051/jpis.2014.44.1.20

Received: Nov. 19, 2013

Accepted: Jan. 7, 2014

eruption (IPE) using occlusal reduction on the periodontal parameters of teeth with moderate chronic periodontitis and traumatic occlusion.

Methods: This study was performed on 40 teeth from 16 subjects. At baseline, 4 weeks after initial periodontal treatment, and 6 months after IPE, clinical and radiographic examinations were performed. The 20 teeth in the test group underwent IPE using occlusal reduction, whereas the 20 control teeth did not undergone occlusal reduction.

Results: All the periodontal parameters were improved by the initial periodontal treatment. The teeth in the test group showed a significantly greater decrease in pocket depth, tooth mobility, and marginal bone loss than did the control group $(P<0.05)$, but there were no significant changes in the attachment level. Significantly greater improvements in all the parameters were observed in the test group after 6 months of IPE compared to 4 weeks after the initial periodontal treatment $(P<0.05)$.

Conclusions: Within the limits of this study, performing initial periodontal therapy combined with IPE using occlusal reduction was shown to be very simple and effective. Moreover, IPE would be helpful in improving periodontal parameters.

Keywords: Occlusal adjustment, Orthodontic extrusion, Periodontitis.

\section{INTRODUCTION}

Dentists generally agree that normal occlusion is a prerequisite for periodontal health. Traumatic occlusion (TO) is defined as an injury resulting in tissue changes within the periodontal attachment apparatus as a result of occlusal forces [1]. Although Svanberg [2] reported that TO does not induce gingivitis, others have insisted that TO may facilitate the progression of periodontitis and cause more alveolar bone resorption [3-5].

Ericsson and Lindhe [5] noted that "the teeth from which the trauma had been removed appeared to have more favorable healing, i.e., gain of connective tissue attachment than the contra lateral hypermobile teeth." The general principle for the initial treatment of periodontitis, in which the etiologic factors are both bacterial plaque and occlusal factors, was to eliminate and control the plaque first, and then deal with the occlusal factors. Therefore, occlusal therapy is a mandatory procedure for treating periodontitis in dental clinics.

Occlusal therapy, in itself, cannot cure gingivitis or periodontitis caused by bacterial activity, but can facilitate plaque control and the management of patients with periodontal disease. Ramfjord and Ash Jr [6] described several modalities of occlusal therapy including orthodontic treatment as part of periodontal treatment. Occlusal adjustment (OA) does
${ }^{*}$ Correspondence:

Ju-Youn Lee

Department of Periodontology, Pusan National University School of Dentistry, 20 Geumo-ro, Beomeo-ri, Mulguem-eup, Yangsan 626-787, Korea

E-mail: heroine@pusan.ac.kr

Tel: +82-55-360-5202

Fax: +82-55-360-5194

This is an Open Access article distributed under the terms of the Creative Commons Attribution Non-Commercial License (http://creativecommons.org/licenses/by-nc/3.0/). 
contribute to occlusal stability, and the forced eruption of teeth with intrabony defects can also reduce the severity of periodontal defects. Orthodontic treatment should always be performed after complete scaling and root planing (SRP) and oral hygiene instruction but before peridontal surgery or restorative procedures [6].

Alveolar bone resorption and periodontal attachment loss are consequences of periodontitis and can compromise the function and aesthetics of periodontal treatment. To overcome this problem, in 1993 Salama and Salama [7] introduced a nonsurgical, noninvasive technique, forced eruption. During extrusion, the crestal bone and supporting soft tissues move vertically. Ingber [8] erupted teeth with one- or two-wall osseous defects, thereby reducing or eliminating the defect and improving the crown-to-root ratio. The belief that forced eruption should alter periodontal defects is clearly based on orthodontic principles.

Passive eruption is a natural eruptive process in response to occlusal wear [9]. Passive eruption can be stimulated artificially by occlusal reduction and this procedure has been termed intentional passive eruption (IPE) in this study. This eruption is less predictable or controllable, but is quite simple and effective when the occlusal reduction is less than $2 \mathrm{~mm}$. Frank et al. [10] reported a successful case using IPE for 5 months to resolve a furcation defect. Occlusal reduction that does not exceed $2 \mathrm{~mm}$, is recommended to deter mesial tipping of the tooth and eliminate the necessity of frequent visits for occlusal reduction [10]. Although occlusal therapy including $\mathrm{OA}$ is essential for initial periodontal therapy, many practitioners face difficulty in the differential diagnosis for TO and application of IPE. Moreover, despite frequently good clinical results reported for OA including IPE, most have been case reports. Therefore, the purpose of this study was to explore the effectiveness of IPE, a type of occlusal reduction, to improve periodontal health and tooth mobility.

\section{MATERIALS AND METHODS}

This study was approved by the Institutional Review Board of Pusan National University Hospital (PNUHIRB 2009109), and was performed in accordance with the Declaration of Helsinki (as revised in Tokyo 2004). Written informed consent was obtained from all subjects.

\section{Subjects}

Forty teeth from 16 patients (10 males, 6 females; mean age, $46 \pm 7$ years) were evaluated. All the patients were recruited at the Department of Periodontology, Pusan National University Hospital. The inclusion criteria were a tooth that had been diagnosed with moderate chronic periodontitis and TO simultaneously. Moreover, the teeth examined had slight tooth mobility and an infrabony defect less than $3 \mathrm{~mm}$.

Patients were excluded for the following reasons: (1) presence of systemic disease; (2) having undergone periodontal treatment $<1$ year before the study; (3) multirooted teeth; (4) teeth with no mo- bility or vertical mobility; (5) teeth restored by prosthesis or heavy restoration.

\section{Treatment protocol}

The initial periodontal treatment including oral hygiene instruction, SRP, and OA was performed in all of the subjects. During the initial periodontal therapy, all the centric occlusion was maintained. After 4 weeks of the initial treatment, the teeth were divided randomly into the test (20 teeth) and the control group (20 teeth). IPE was performed in the test teeth and additional IPE was not performed in the control teeth. Clinical and radiographic examinations were performed at baseline and 4 weeks after initial treatment. After 6 months of IPE, all of the assessments were repeated. The periodontal treatment procedure, periodontal examination, and radiographic evaluation were performed separately, each by one of 3 periodontists.

The exact method of IPE used was as follows: All the occlusal reduction was performed using a high-speed diamond bur. While centric occlusion was maintained, routine OA was carried out. On the other hand, IPE was performed until the target tooth was completely out of the occlusion and repeated every 4-6 weeks to guarantee space for passive eruption and to allow the formation of reparative dentin. IPE was stopped when the tooth mobility decreased or the patient's discomfort disappeared and the total amount of occlusal reduction was less than $2 \mathrm{~mm}$. During the IPE, opposing teeth were stabilized via splinting to prevent extrusion of opposing teeth. The amount of occlusal reduction was measured by biting impression material (Futar D., Kettenbach, Germany) and measuring the thickness using digital calipers.

\section{Clinical measurement}

The following indices were measured to assess the periodontal status and oral hygiene level: (1) plaque index (PI) [11]; (2) gingival index [12]; (3) probing depth (PD); (4) clinical attachment level (CAL); (5) bleeding on probing (BOP); (6) width of keratinized gingiva (KG); and (7) tooth mobility (Periotest value, PTV).

The PD, CAL, and BOP were measured at 4 sites of each tooth (mesiobuccal, midbuccal, distobuccal, and lingual). The PD and CAL were measured using a Williams probe (Hu-Friedy, Chicago, IL, USA) by rounding up to the nearest millimeter, and the mean value per tooth was used. The KG was measured from the midbuccal gingival margin to the mucogingival junction parallel to the long axis of the teeth. The PTV was measured 5 times for each tooth using the Periotest (Siemens AG, Bensheim, Germany), by which the teeth were impacted pulsatively, that is, for an interval of less than $1 \mathrm{~ms}$. PTV is a dynamic equivalent to the damping properties of the tooth and its periodontium in the vestibule-oral direction. The final value was defined as the average excluding the highest and lowest value.

\section{Radiographic measurement}

The radiographic evaluations were performed at baseline, 4 weeks after initial periodontal therapy, and 6 months after IPE. Periapical 
radiographs were taken using the standard paralleling technique. Each radiograph was digitized by computer scanning and measured using an image analysis software program (AxioVision, Carl Zeiss Microlmaging $\mathrm{GmbH}$, Jena, Germany). The marginal bone loss was measured from the cementoenamel junction to the alveolar bone crest, parallel to the root surface, and separately at the mesial and distal tooth surface by a single examiner. The measurements were corrected based on the tooth width and averaged.

\section{Statistical analysis}

A statistical software package, SPSS ver. 14.0 (SPSS Inc., Chicago, IL, USA), was used for statistical analyses, with the level of significance of $P<0.05$. An independent $t$-test was used to compare the clinical parameters between the two groups at baseline, 4 weeks after initial periodontal therapy, and 6 months after IPE. The differences between 4 weeks after initial periodontal therapy and 6
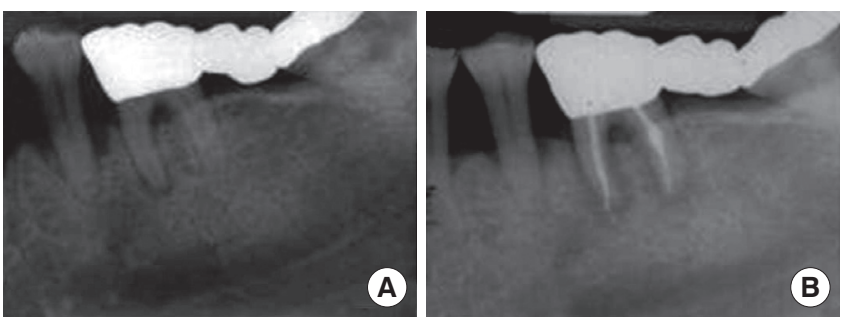

Figure 1. Periapical radiograph of a left mandibular second premolar. (A) A radiograph at baseline showing severe periodontal attachment loss, an intrabony defect, and a widened periodontal ligament space. (B) A radiograph after 6 months of intentional passive eruption showing decreased periodontal ligament space and resolution of the intrabony defect. Note the flatness of the cuspal inclination and the change of the position of the root apex due to intentional passive eruption. months after IPE were analyzed using a paired $t$-test.

\section{RESULTS}

All of the teeth that were examined healed uneventfully and the patients did not complain of any complications or discomfort. A resolution of intrabony defect and distinct lamina dura was noticeable in the radiograph (Fig. 1). The two groups showed similar clinical parameters at baseline, except for PI and marginal bone loss (Table 1). Four weeks after initial periodontal therapy including $O A$, the clinical parameters had improved $(P<0.05)$, except for the marginal bone loss in the control group and $K G$ in both groups (Table 1).

\section{Comparison of the clinical parameters after 6 months of IPE}

The mean occlusal reduction was $0.79 \mathrm{~mm}$ and there were no symptoms associated with dental hypersensitivity. After 6 months of IPE, significant differences in PD, PTV, and $K G$ were observed between the 2 groups $(P<0.05)$ (Table 1). Compared to 4 months after initial therapy, all the clinical parameters were improved in the test group $(P<0.05)$, but there were no significant differences in the control group (Table 1).

\section{DISCUSSION}

SRP was reported to be the best single therapy option available for the treatment of moderate periodontitis and had positive effects on the clinical parameters of PD, CAL, and tooth mobility [13-15]. Adjunctive treatments continue to be explored but there are still no stand-alone replacement therapies for mechanical de-

Table 1. Comparison of the clinical parameters between the test and control group.

\begin{tabular}{|c|c|c|c|c|c|c|}
\hline \multirow[b]{2}{*}{ Variable } & \multicolumn{3}{|c|}{ Test } & \multicolumn{3}{|c|}{ Control } \\
\hline & Baseline & $\begin{array}{l}4 \text { Weeks after } \\
\text { initial therapy }\end{array}$ & 6 Months after IPE & Baseline & $\begin{array}{l}4 \text { Weeks after } \\
\text { initial therapy }\end{array}$ & 6 Months after IPE \\
\hline Plaque index & $1.25 \pm 0.55$ & $0.60 \pm 0.50^{b)}$ & - & $1.75 \pm 0.44^{\mathrm{a})}$ & $0.60 \pm 0.50^{\mathrm{bl}}$ & - \\
\hline Gingival index & $1.10 \pm 0.64$ & $0.60 \pm 0.68^{b)}$ & - & $1.40 \pm 0.50$ & $0.50 \pm 0.51^{b)}$ & - \\
\hline Probing depth (mm) & $3.51 \pm 0.85$ & $2.83 \pm 0.72^{\mathrm{b})}$ & $2.37 \pm 0.36^{\mathrm{d})}$ & $3.99 \pm 1.26$ & $2.85 \pm 0.61^{\mathrm{b})}$ & $2.86 \pm 0.61^{c)}$ \\
\hline $\mathrm{CAL}(\mathrm{mm})$ & $3.73 \pm 1.02$ & $3.36 \pm 0.72^{\mathrm{b})}$ & $2.98 \pm 0.44^{d}$ & $4.17 \pm 1.67$ & $3.48 \pm 1.22^{b)}$ & $3.36 \pm 1.04$ \\
\hline Bleeding on probing & $0.85 \pm 0.37$ & $0.55 \pm 0.51^{b)}$ & - & $1.00 \pm 0.00$ & $0.60 \pm 0.50^{b)}$ & - \\
\hline Periotest value & $21.41 \pm 6.20$ & $20.13 \pm 6.03^{b)}$ & $9.88 \pm 2.28^{\mathrm{d})}$ & $22.02 \pm 5.80$ & $19.84 \pm 5.70^{b)}$ & $19.80 \pm 5.58^{c)}$ \\
\hline Keratinized gingiva (mm) & $2.10 \pm 0.72$ & $2.25 \pm 0.32$ & $2.65 \pm 0.75^{d)}$ & $1.83 \pm 0.75$ & $1.65 \pm 0.46$ & $1.82 \pm 0.75^{c)}$ \\
\hline Marginal bone loss (mm) & $5.12 \pm 1.23$ & $5.10 \pm 1.22^{b)}$ & $4.52 \pm 1.17^{d)}$ & $4.18 \pm 0.91^{a)}$ & $4.15 \pm 0.90$ & $4.13 \pm 0.89$ \\
\hline
\end{tabular}

Values are presented as mean \pm standard deviation

CAL: clinical attachment level, IPE: intentional passive eruption.

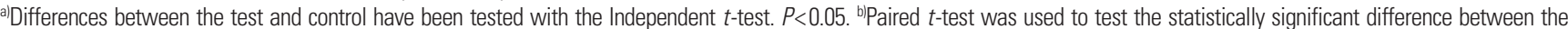
baseline and after 4 weeks of initial periodontal therapy. $P<0.05$. ${ }^{c}$ Independent $t$-test was used to test the statistically significant difference between test and control group at 6 months after intentional passive eruption. $P<0.05$. ${ }^{d}$ Paired $t$-test was used to test the statistically significant differences between 4 weeks after initial periodontal therapy and 6 months after intentional passive eruption. $P<0.05$. 
bridement. SRP is a foundation therapy for many adjunctive treatments. In this study, overall improvements in the clinical parameters were observed after initial periodontal treatment, as reported previously [13-15].

TO is defined and diagnosed based on the histological changes in the periodontal supporting structure. As it is impractical for a dentist to diagnose TO clinically, clinicians must rely on the clinical signs and symptoms of potential TO. The typical symptoms are pain or discomfort including periodontal pain, dental hypersensitivity, loose teeth, pathologic migration of the teeth, and so on [16]. On the other hand, it is very difficult for dentists to distinguish between TO and periodontal inflammation by symptoms only. The regeneration of periodontal defects is almost completely possible when periodontal inflammation and TO are removed at the same time [17]. In this study, the periodontist tried to diagnose TO from the clinical signs, symptoms, and radiographic findings, and to remove the occlusal interference during the initial treatment. After four weeks of initial treatment including OA, significant improvements in most of the clinical parameters were observed except for marginal bone loss and KG in accordance with other studies [1315]. This data can be explained as follows: 4 weeks is too brief a period to anticipate a change in the bone level and the $\mathrm{KG}$ is stable due to a lack of additional changes in the vertical tooth position.

Everett and Baer [18] introduced the idea of IPE, defined a protocol of IPE, and reported good clinical results. Briefly, the involved tooth with an infrabony defect is completely removed from the occlusion. The occlusal grinding should be repeated every 3 months until a satisfactory bone contour is obtained. On the other hand, in this study, the occlusal reduction was repeated every 4 to 6 weeks because the periodontal status of the targeted teeth was more severe and mobile than those reported by Everett and Baer [18] and all of the teeth examined were single-rooted teeth. This should have provided sufficient time and space for the passive eruption of the teeth.

A comparison of the clinical parameters after 6 months of IPE with the values at 4 weeks after the initial treatment showed that all of the clinical parameters had improved $(P<0.05)$. Moreover, a comparison of the clinical parameters between the 2 groups after 6 months of IPE revealed IPE to have had additional effects on PD, $P T V$, and $K G$ in the test group $(P<0.05)$.

Tooth mobility is an indicator of the status of the periodontium and is one of the most important factors for diagnosing the prognosis of teeth and determining a appropriate treatment plan. In this study, the tooth mobility was measured using a Periotest for its reliability and reproducibility. The PTV is strongly correlated with the amount of alveolar bone [19]. The amount of occlusal reduction during IPE was $0.79 \mathrm{~mm}$, and the increase in the $\mathrm{KG}$ was approximately $50 \%$ of the occlusal reduction. In a monkey model, Kajiyama et al. [20] reported the following: (1) the free gingiva moved approximately 90\% and the attached gingiva moved approximately $80 \%$ as far as the teeth were extruded; (2) the width of the attached gingiva increased as the teeth were extruded; and
(3) the mucogingival junction was positioned the same before and after forced eruption. Therefore, these results support the present findings in that the KG increased after IPE. After 6 months of IPE, the marginal bone loss was similar in the 2 groups, whereas at baseline, the marginal bone loss had been greater than in the control group $(P<0.05)$. These findings suggest that the marginal bone loss was decreased by IPE.

Although the aesthetic needs of patients have increased, aesthetic defects due to periodontitis are inevitable. Many case reports of the treatment of aesthetic defects using forced eruption have been reported $[21,22]$. The resorbed alveolar bone causes compression of the periodontal membrane, and new bone is deposited whenever there is stretching force acting upon the alveolar bone [23]. Another drawback of periodontitis is the poor crown to root ratio, and IPE, which is a type of forced eruption, may improve the crown to root ratio and prognosis of the periodontal health [8]. In this study, the periodontal parameters were improved by SRP and OA in both groups and additional IPE showed an improvement in the periodontal indexes for treating teeth with mobility and periodontal intrabony defects. Furthermore, because the application of IPE is very simple and safe, IPE would be helpful in improving periodontal parameters.

\section{CONFLICT OF INTEREST}

No potential conflict of interest relevant to this article was reported.

\section{ACKNOWLEDGEMENTS}

This study was supported by a 2-year Research Grant of Pusan National University.

\section{ORCID}

Ju-Youn Lee http://orcid.org/0000-0002-0772-033X

\section{REFERENCES}

1. Hallmon WW. Occlusal trauma: effect and impact on the periodontium. Ann Periodontol 1999;4:102-8.

2. Svanberg G. Influence of trauma from occlusion on the periodontium of dogs with normal or inflamed gingivae. Odontol Revy 1974;25:165-78.

3. Polson AM, Meitner SW, Zander HA. Trauma and progression of marginal periodontitis in squirrel monkeys. III Adaption of interproximal alveolar bone to repetitive injury. J Periodontal Res 1976; 11:279-89.

4. Lindhe J, Ericsson I. The influence of trauma from occlusion on reduced but healthy periodontal tissues in dogs. J Clin Periodontol 1976;3:110-22.

5. Ericsson I, Lindhe J. Lack of significance of increased tooth mo- 
bility in experimental periodontitis. J Periodontol 1984;55:447-52.

6. Ramfjord SP, Ash MM Jr. Significance of occlusion in the etiology and treatment of early, moderate, and advanced periodontitis. J Periodontol 1981;52:511-7.

7. Salama $H$, Salama M. The role of orthodontic extrusive remodeling in the enhancement of soft and hard tissue profiles prior to implant placement: a systematic approach to the management of extraction site defects. Int J Periodontics Restorative Dent 1993;13:312-33.

8. Ingber JS. Forced eruption. I. A method of treating isolated one and two wall infrabony osseous defects-rationale and case report. J Periodontol 1974;45:199-206.

9. Steedle JR, Proffit WR. The pattern and control of eruptive tooth movements. Am J Orthod 1985;87:56-66.

10. Frank CA, Pearson BS, Booker BW. Orthodontic eruption of furcainvolved molars. Compend Contin Educ Dent 1995;16:664, 666, 668 passim.

11. Silness J, Loe H. Periodontal disease in pregnancy. II. Correlation between oral hygiene and periodontal condtion. Acta Odontol Scand 1964;22:121-35.

12. Löe $H$. The gingival index, the plaque index and the retention index systems. J Periodontol 1967;38:610-6.

13. Kerry GJ, Morrison EC, Ramfjord SP, Hill RW, Caffesse RG, Nissle $\mathrm{RR}$, et al. Effect of periodontal treatment on tooth mobility. J Periodontol 1982;53:635-8.

14. Pihlstrom BL, McHugh RB, Oliphant TH, Ortiz-Campos C. Comparison of surgical and nonsurgical treatment of periodontal disease: a review of current studies and additional results after $61 / 2$ years. J Clin Periodontol 1983;10:524-41.

15. Suvan JE. Effectiveness of mechanical nonsurgical pocket therapy. Periodontol 2000 2005;37:48-71.

16. Ramfjord S, Ash M. Occlusion. Philadelphia: WB Saunders; 1983.

17. Kantor M, Polson AM, Zander HA. Alveolar bone regeneration after removal of inflammatory and traumatic factors. J Periodontol 1976;47:687-95.

18. Everett FG, Baer PN. A preliminary report on the treatment of the osseous defect in periodontosis. J Periodontol 1964;35:429-35.

19. Demirel K, Gur H, Meric H, Sevuk C. Damping characteristics of teeth with periodontal breakdown: correlation of mobility meter values with bone and attachment loss. J Periodontol 1997;68: 166-71.

20. Kajiyama K, Murakami T, Yokota S. Gingival reactions after experimentally induced extrusion of the upper incisors in monkeys. Am J Orthod Dentofacial Orthop 1993;104:36-47.

21. Mankoo T, Frost L. Rehabilitation of esthetics in advanced periodontal cases using orthodontics for vertical hard and soft tissue regeneration prior to implants: a report of 2 challenging cases treated with an interdisciplinary approach. Eur J Esthet Dent 2011; 6:376-404.

22. Lin CD, Chang SS, Liou CS, Dong DR, Fu E. Management of interdental papillae loss with forced eruption, immediate implantation, and root-form pontic. J Periodontol 2006;77:135-41.

23. Graber TM, Adams PE. Current orthodontic concepts and techniques. Philadelphia: Saunders; 1969. 